\title{
New Frontiers in Placenta Tissue Imaging
}

\author{
Authors: \\ Christopher D. Nguyen, ${ }^{1}$ Ana Correia-Branco, ${ }^{2,3}$ Nimish Adhikari, ${ }^{4}$ Ezgi \\ Mercan, ${ }^{5}$ *Srivalleesha Mallidi, ${ }^{1}$ Mary C. Wallingford ${ }^{2,3,6}$ \\ 1. Department of Biomedical Engineering, Tufts University, Medford, \\ Massachusetts, USA \\ 2. Mother Infant Research Institute, Tufts Medical Center, Boston, Massachusetts, \\ USA \\ 3. Molecular Cardiology Research Institute, Tufts Medical Center, Boston, \\ Massachusetts, USA \\ 4. Department of Computer Science, Tufts University, Medford, Massachusetts, USA \\ 5. Craniofacial Center, Seattle Children's Hospital, Seattle, Washington, USA \\ 6. Obstetrics and Gynecology, Tufts University School of Medicine, Boston, \\ Massachusetts, USA \\ *Correspondence to srivalleesha.mallidi@tufts.edu and \\ mwallingford@tuftsmedicalcenter.org
}

Disclosure:

The authors have declared no conflicts of interest.

Received:

Accepted:

Keywords:

Citation:
23.12 .19

01.07.20

Computer vision, hypoxia imaging, metabolic imaging, morphomics, placenta, structural imaging, vascular imaging.

EMJ Radiol. 2020;1[1]:54-62.

\section{Abstract}

The placenta is a highly vascularised organ with unique structural and metabolic complexities. As the primary conduit of fetal support, the placenta mediates transport of oxygen, nutrients, and waste between maternal and fetal blood. Thus, normal placenta anatomy and physiology is absolutely required for maintenance of maternal and fetal health during pregnancy. Moreover, impaired placental health can negatively impact offspring growth trajectories as well as increase the risk of maternal cardiovascular disease later in life. Despite these crucial roles for the placenta, placental disorders, such as preeclampsia, intrauterine growth restriction, and preterm birth, remain incompletely understood. Effective noninvasive imaging and image analysis are needed to advance the obstetrician's clinical reasoning toolkit and improve the utility of the placenta in interpreting maternal and fetal health trajectories. Current paradigms in placental imaging and image analysis aim to improve the traditional imaging techniques that may be time-consuming, costly, or invasive. In concert with conventional clinical approaches such as ultrasound, advanced imaging modalities can provide insightful information on the structure of placental tissues. Herein, the authors discuss such imaging modalities; their specific applications in structural, vascular, and metabolic analysis of placental health; and emerging frontiers in image analysis research in both preclinical and clinical contexts. 


\section{INTRODUCTION}

The placenta plays numerous critical roles during pregnancy, from modulating adaptations of maternal cardiovascular, skeletal, and endocrine systems, to controlling transport of nutrients, oxygen, and waste between maternal and fetal circulations. Impaired development and/or function of the placenta can result in adverse maternal and fetal clinical outcomes. With respect to the development of advanced diagnostic tools and treatment options that can potentially inform and improve clinical practices, noninvasive biomedical imaging of the placenta provides a promising approach. Herein, the authors discuss imaging modalities that can be used to generate insight into anatomical structure, vascular physiology, and metabolism of the placenta (Figure 1) $\cdot^{1-5}$ For example, multiple modalities are now available that can be used to study placental blood flow, maternal-placental and fetoplacental vasculature, placental microcirculation, the spatial pattern of fetoplacental density, placental sufficiency, intrauterine hypoxia, placental oxygenation, and haemoglobin oxygenation in normal and pathological conditions. Additionally, the increased value and analytic depth of multiparametric three-dimensional (3D) computational image analysis methods that accompany these imaging modalities are discussed in detail. Finally, in the last section of this review the authors discuss how advanced computational approaches such as computer vision, automation, and deep learning strengthen the power of preclinical and clinical biomedical imaging data.

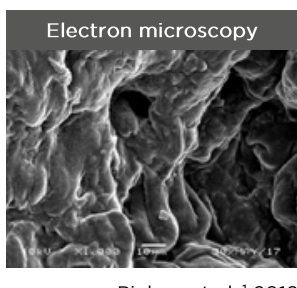

Pielesz et al.,1 2019

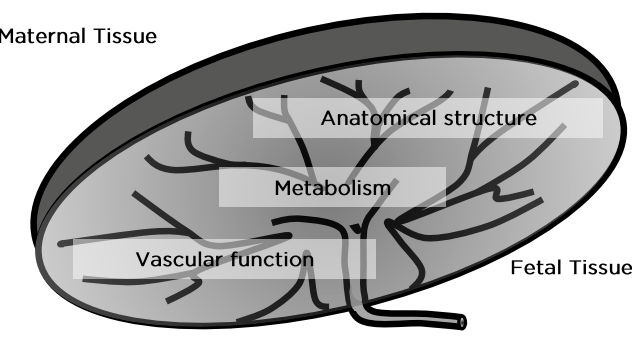

Aughwane et al., 42019
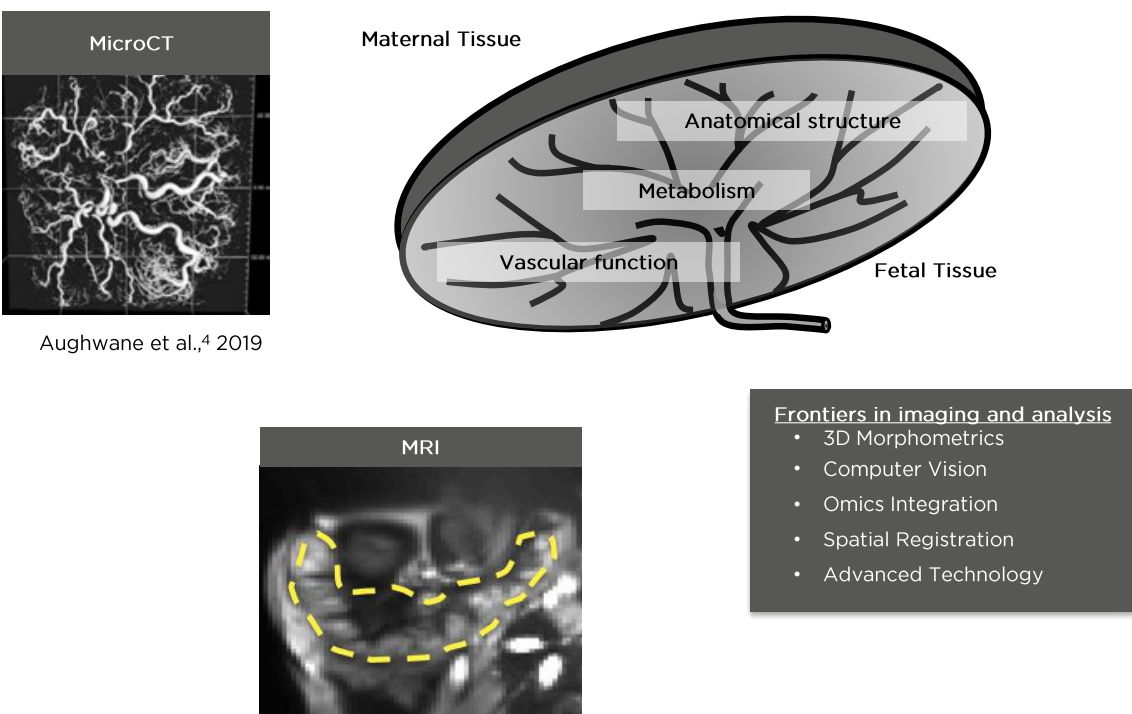

Luo et al.,5 2017

Figure 1: Examples of imaging modalities that can be used to generate insight into anatomical structure, vascular, and metabolic function of the placenta. ${ }^{1-5}$ 


\section{NEW FRONTIERS IN CLINICAL IMAGING}

\section{Structure}

Detection of abnormalities in placental structure is clinically important because it allows clinicians to diagnose symptoms early in the pregnancy and develop individual treatment responses. ${ }^{4}$ These structures include the boundaries of different placental compartments, the size and volume of the placenta and extraembryonic tissues, and the overall location of these structures with respect to one another. Microfocus CT (microCT) is a fast-growing imaging technique that can provide highresolution, large field-of-view 3D morphological structures of the placenta. Its use in quantitative analysis of the fetoplacental vascular tree and the feasibility for obtaining this information on human samples with the aim of understanding complications of intrauterine growth restriction (IUGR) has been demonstrated ex vivo. ${ }^{6}$ In a complementary ex vivo study, Pratt et al. ${ }^{7}$ compared two contrast agents by their capacity to produce superior image quality for improved measurements of placental vascular density and analysis of the vascular tree structure, and determined MICROFIL ${ }^{\circledR}$ to be the better contrast agent for this application. Despite the capabilities of microCT to generate nondestructive high-resolution images, this modality cannot be used for human placental imaging in vivo because of its use of an ionising source, but it remains an invaluable tool for preclinical or clinical ex vivo quantification of morphology and vascularity.

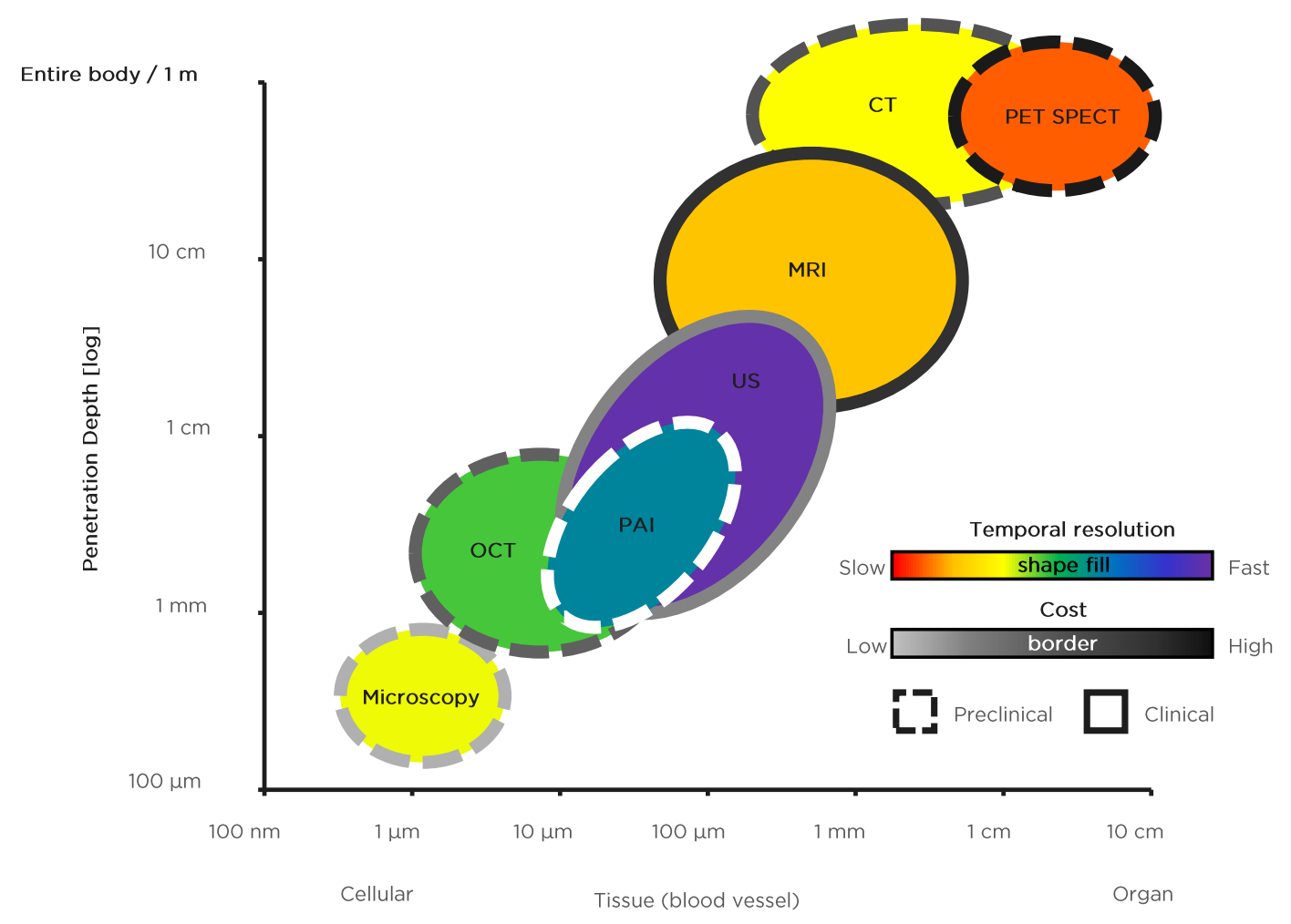

Spatial resolution [log]

Figure 2: Schematic representation of spatial resolution versus penetration depth of various preclinical (dashed border) and clinical (solid border) imaging modalities used for placental and extraembryonic tissue imaging. The temporal resolution is represented by a pseudo-colourmap, with red representing slow and violet representing fast. The cost is represented by a different pseudo-colourmap, with grey representing low cost and black representing high cost. Note that photoacoustic imaging has a dashed white border which represents its primary use in preclinical studies but has yet to be clinically used in any form. Given the absence of clinical applications for photoacoustic imaging, associated costs for this technique are not included. 
Non-ionising techniques such as MRI, which provides better soft-tissue contrast, or ultrasound (US), which provides higher throughput real-time images, are more commonly used in preclinical and clinical studies. Lax et al. $^{8}$ and Teo et al. ${ }^{9}$ provided three easily identifiable in vivo features useful in diagnosing placental invasion in T2weighted MRI images: 1) lower uterine bulging; 2) heterogenous placental signal intensity; and 3) dark linear intraplacental bands. However, MRI is best used as an adjunct to US, particularly when the US resolution is diminished as a result of obesity or a posteriorly situated placenta. Furthermore, as shown in Figure 2, US is lowcost compared with MRI and is advantageous for longitudinal studies to monitor fetal growth and changes in placenta function. In a retrospective in vivo US study, Rac et al..$^{10}$ developed a predictive equation of placental invasion termed placenta accreta index, which relies on the number of prior caesarean deliveries and several US parameters including smallest myometrial thickness, lacunar spaces, and the presence of bridging vessels. Additionally, alongside placental compartments, US can be used to image the umbilical cord insertion point. For example, Wax et al." determined that cord insertion-to-placental edge distance could be used to diagnose marginal cord insertion and risk for prelabour rupture of membrane, preterm prelabour rupture of membrane, extreme umbilical cord lengths, and spontaneous preterm birth as described by Ebbing et al. ${ }^{12}$ With respect to structural assessments, US followed by MRI is the preferred methodology overall to obtain structural tissue contrast, but further studies directly comparing the specificity and sensitivity of the two are needed.

\section{Vascular Function}

Normal fetal growth requires sufficient oxygen and nutrient delivery through the placenta. Therefore, quantification of maternal-fetal perfusion and vascular function is crucial in diagnosing placental abnormalities. Historically, preclinical ex vivo microscope analysis has dominated interpretation of placental vascular development and function. For example, Coan et al. $^{13}$ performed stereological analysis on mouse placentas to quantify the development of the labyrinth zone, which serves as the nutrient transport barrier and mediator between the maternal and fetal circulatory systems. Furthermore, these methods support predictions of the diffusion capacity of oxygen. In general, postmortem microscope analysis can provide high-resolution images for modelling of placental vascular function, but to clearly predict the health of the fetus and understand the functional interdependence of the maternal-placental-fetal triad, longitudinal monitoring is needed because ex vivo microscopic analysis is not sufficient.

Functional MRI can address the need for longitudinal monitoring by providing online monitoring of blood flow using blood oxygen level dependent (BOLD). In a study by Sørensen et al.,14 dark and bright areas corresponding to the fetal and maternal side, respectively, in BOLD placental images during normoxia and hyperoxia were compared. BOLD signal increased as darker areas became brighter, which is indicative of increased placental oxygenation. Thus, a reduced or constant placental BOLD signal during hyperoxia may be associated with impaired placental function and poor neonatal outcome. Furthermore, BOLD images of the placenta acquired over time can be used to generate timeto-plateau maps, which can aid in identifying regional heterogenous oxygenation in the placenta. ${ }^{5}$ This promising biomarker had relatively homogenous values near zero under normoxia conditions, while placentas under maternal hyperoxia displayed inhomogeneous delayed values. Indeed, multiple studies concluded that maternal hyperoxia induces the hyperoxic BOLD effect, which is an increase in placental signal intensity in T2-weighted images as reviewed by Sørensen et al. ${ }^{15}$ In pregnancies complicated by IUGR, similar effects were observed with a significantly increased BOLD effect compared with control cases. However, the authors noted that the BOLD effect is a relative measurement and that an increase in BOLD signal may originate from a reduced T2-signal baseline rather than an increased absolute signal. A baseline T2signal may therefore be a sufficient initial clinical biomarker for detecting dysfunctional placentas.

Online monitoring of blood flow can additionally be accomplished using arterial spin labelling (ASL) MRI. Using pseudocontinuous ASL MRI, Liu et al. ${ }^{16}$ generated four perfusion-related parameters: placental volume, placental blood flow (PBF), high PBF (hPBF), and relative hPBF. In a longitudinal study, patients with ischaemic 
placentas showed significant decreases in $\mathrm{hPBF}$ and relative hPBF values compared with controls, suggesting its promise as a biomarker for predicting placental ischaemia. By monitoring placenta perfusion, diseases such as IUGR or congenital heart disease (CHD) have been linked to dysfunctional placentas. For example, placental perfusion velocity-selective ASL (VSASL) MRI measurements have value for predicting fetal $\mathrm{CHD}^{17}$ In fetal CHD pregnancies, variation in regional placental perfusion significantly increased while global placental perfusion significantly decreased with advancing gestational age. Overall, functional variants of MRI can quantify maternal-fetal perfusion. However, MRI variants that rely on the aid of exogenous contrast agents (contrast enhanced [CE] MRI), such as gadolinium, are not approved for human use because of its dissociation into toxic form. ${ }^{18}$ Therefore, similar to structural MRI, functional MRI, but not CE MRI, may be used as an adjunct to functional US to clinically evaluate vascular function.

CE US (CEUS) enables imaging of structures with low perfusion by employing the use of gas-filled, lipid-encapsulated microbubbles to increase contrast because of the acoustic impedance mismatch from surrounding tissue. In an in vivo study by Roberts et al. ${ }^{19}$ to assess the safety of CEUS for studying placental perfusion in rhesus macaques and human subjects, placental structural damage was not observed after multiple CEUS examinations. Additional results of the examinations in human subjects demonstrated visualising placental perfusion in as early as 11 weeks. ${ }^{19}$ As a clinical continuation of this work, Roberts et al. ${ }^{20}$ used CEUS to assess maternal blood flow into the intervillous space and reported the detection of maternal perfusion through the spiral arteries in as early as 6 weeks. This suggests that remodelling of extravillous trophoblast cell clusters to reduce flow resistance into the intervillous space may be a progressive process that occurs earlier than previously suspected. ${ }^{20}$ Doppler US imaging is traditionally used to quantify the maternal-fetal blood flow. ${ }^{21}$ Uterine artery Doppler, a combination of pulsed and colour Doppler features, can be used to measure the human maternal-fetal blood flow to show that most early IUGR cases display abnormal waveforms, which is associated with coexisting preeclampsia and maternal-placental vascular malperfusion. ${ }^{22}$ Furthermore, uterine artery Doppler in combination with multiple maternal serum markers can be used to predict the development of preeclampsia. ${ }^{23,24}$ With respect to high-risk pregnancies, Doppler US has been supported to reduce the risk of perinatal deaths with potentially fewer obstetric interventions. ${ }^{25}$ Further studies correlating fetal development to maternal-fetal blood flow are required and could provide invaluable insights into developing therapies for placental malfunction.

\section{Metabolism}

Irregularities in cellular metabolism have shown to be surrogate markers of disease. In the case of obesity, Calabuig-Navarro et al. ${ }^{26}$ have proposed that the metabolic capacity of the placenta plays a central role in regulating fetal adiposity and conferring disease risk to offspring. Indeed, placental fatty acid esterification and mitochondrial fatty acid $\beta$-oxidation associate with markers of fetal adiposity in the context of maternal obesity. ${ }^{26}$ Mechanistically, this may relate to the interaction between fatty acid oxidation, mitochondrial function, and oxidative stress. ${ }^{27}$ Thus, it may be insufficient to strictly perform structure and vascular imaging. Metabolic imaging is a relatively new field that observes and tracks changes in metabolic pathways that may be linked to various clinical conditions. Quantification of the placental metabolic capacity, in addition to structure and vascular function, can lead to a better understanding of the interaction between placental function and fetal development. For example, Austin et al. ${ }^{28}$ elaborated on the importance of evaluating the spatial distribution of elemental metal species and their role in metabolic processes, such as oxygen transportation and mitochondrial function. Deficiencies in these essential metals and/or long-term exposure to exogenous heavy metals are associated with the negative health of a child. A predominant methodology that can be used to obtain placental metabolic data is proton magnetic resonance spectroscopy ( $\left.{ }^{1} \mathrm{H}-\mathrm{MRS}\right)$. In a clinical in vivo study, Denison et al..$^{29}$ used ${ }^{1} \mathrm{H}-\mathrm{MRS}$ to measure the choline-lipid ratio in severe cases of placental insufficiency that were associated with IUGR. Current evidence suggests that a reduction in placental choline peaks is associated with poor cellular turnover; therefore, the cholinelipid ratio may be used as a novel biomarker 
to detect impaired placental function. Song et al. ${ }^{30}$ performed a similar study in which they proposed to combine the choline-lipid ratio with apparent diffusion coefficient values from diffusion-weighted MRI to enhance the sensitivity and specificity of detecting IUGR. Compared with the data presented by Denison et al., the lipid peaks in the present study significantly increased with IUGR severity. The discordance in these findings may relate to the population-based differences in the severities of IUGR in these two studies.

To further understand the involvement of metabolism in placental abnormalities, variants of Raman spectroscopy, a label-free method based on Raman scattering that provides the molecular structure of the sample, can be used. Thus far these studies have been largely restricted to ex vivo studies. Pielesz et al." tested the application of Fourier transform Raman spectroscopy to identify spectroscopic biomarkers or specific frequency ranges that may accurately indicate the transport efficiency governed by syncytiotrophoblast. The most notable region observed was the $950-750 \mathrm{~cm}^{-1}$ frequency range, which represented changes in molecular structure to differentiate healthy from pathological tissue. Using micro-Raman spectroscopy, Chen et al. ${ }^{31}$ studied the metabolic variation in preeclamptic placentas. Compared with normal placentas, preeclamptic placentas displayed higher amplitudes at peaks assigned to the tryptophan indole ring and phenylalanine, which suggest a significant reduction in the ordered structures of the proteins as well as damage to the amino acid side chains. Overall, these studies support the notion that Raman spectroscopy is effective in differentiating healthy from pathological placental tissue and therefore can be used to increase the diagnostic accuracy of placental insufficiency.

\section{NEW FRONTIERS IN PRECLINICAL IMAGING}

One major need in placental imaging is to understand the dynamic changes in blood oxygen saturation and vascular perfusion in vivo without the use of exogenous contrast agents. To address this, several advances have been made towards photoacoustic imaging (PAI), an imaging modality that combines US with the high-molecular sensitivity of optical imaging to measure changes in oxygen saturation by exploiting the different absorption spectrums of oxy- versus deoxyhaemoglobin in the placenta. ${ }^{32}$ Extracting the saturation level involves fitting the PA intensity to the optical absorption of haemoglobin at various excitation wavelengths. ${ }^{33}$ Using PAl in a rat and mouse study, Lawrence et al. $^{34}$ and Yamaleyeva et al.,35,36 respectively, measured placental hypoxia and evaluated its association with the progression of preeclampsia or IUGR due to ischaemia. Key challenges that must be addressed prior to clinical translation of PAI are limited light delivery to deep fetal tissues and safety limits of nanosecond pulsed radiation exposure for developing tissues.

In addition to translating technology for clinical trials, preclinical studies support investigation of the relationship between placental biology and embryonic development through the use of models with short gestation periods and homogeneous genetic traits. Yadav et al. ${ }^{37}$ used dynamic CE MRI to quantitatively study the gestational age-dependent perfusion changes and vascular diameters in the regional compartments of normal murine placenta. With advancing gestation, the maternal central canal diameter increases, which may contribute to an increase in placental perfusion. In a separate longitudinal study by Krishnamurthy et al., ${ }^{38}$ T2-weighted MRI signals were measured with advancing gestational age. With advancing gestation, T2 relaxation times decreased in both high and low perfusion zones with absolute T2 signal values in low perfusion zones matching the whole placenta.

To address aspects of placenta biology that are specific to primates, extensive, ground-breaking research has been performed on nonhuman primate models, such as the rhesus macaque, a more clinically relevant translational animal model establishing the maternal-fetal-placental unit function during pregnancy. ${ }^{39}$ In a placental perfusion study of rhesus macaques, BOLD and dynamic CE MRI placental images were compared to create a model connecting the observed T2-weighted images to a modified BOLD signal parameter that relates the intervillous blood flow and oxygen exchange with the fetal vasculature. ${ }^{40}$ Furthermore, with the aid of contrast agents, Lo et al. ${ }^{41}$ used Doppler US with dynamic CE MRI to detect IUGR in nonhuman primate models. T2-weighted MRI and anisotropic 
water diffusion images of the fetal brain in utero were acquired to characterise the aberrant effects of IUGR on fetal neurodevelopment, such as reduced brain volume. Several new contrast agents are being developed to obtain specific molecular information for these imaging techniques, such as MRI, US, and optical techniques; however, these agents still need to be evaluated thoroughly prior to clinical translation. ${ }^{42-44}$ Overall, longitudinal preclinical studies play a crucial role in translating these technological advancements to the clinic.

\section{FUTURE OF IMAGING}

\section{Computer Vision}

Computer vision techniques provide a particularly promising new frontier for the evaluation of placental physiology and structurefunction relationships. These techniques make use of certain features to numerically describe physical properties of the placenta, which, once determined, can be used to test for correlation between clinical characteristics of the pregnancy as well as clinical maternal-fetal outcomes. For example, placental maturity and gestational age have been tested in US and MRI images with positive results. Specifically, grey-level cooccurrence matrix texture features, known as Haralick texture features, have been used to show an increase in placental heterogeneity throughout the gestational age. ${ }^{45,46}$

Image analysis is increasingly combined with machine learning techniques that provide prediction models for examining correlation between clinical characteristics and outcomes. A multitude of machine learning and clustering methods, including Naïve Bayes, $K$ nearest neighbors, k-means, and multilayer perceptron, have been successfully used alongside texture analysis techniques for MRI and US images. For example, Romeo et al. ${ }^{47}$ used machine learning methods with MRI-derived texture analysis features to assess the presence of placenta accreta spectrum in patients with placenta praevia with an accuracy up to $98 \%$. Using an alternative segmentation approach, Gupta et al. ${ }^{48}$ applied wavelet decompositionbased conditional random fields to successfully develop segmentation methods for US images of the fetus. These tools could potentially be applied to placenta segmentation using proper constraints and choice of the training set features.

\section{Morphomics}

Morphomics is a recently coined term that involves quantitative measurements generated via image analysis software and scientific visualisation tools. These analytic morphomics aid in personalised care by stratifying the image analysis data with respect to disease status. Compared with platform-dependent and specialised commercial software used in clinical settings, a wide range of open-source, free, and customisable software tools are available for morphomic analysis. ImageJ is an open-source software that became popular as a result of its large repository of plugins and macros, including 3D viewer plugins, developed by the userbase for different tasks and image formats. ${ }^{49}$ Quantitative analysis of villi anatomy in the placenta, molecule transportation across the maternal-fetal interface, and analysis of volumetric data to study placental microcirculation, such as the spatial pattern of fetoplacental vascular density, is possible with these tools. ${ }^{3,7,50-52}$

Insight Segmentation and Registration Toolkit (ITK) provides software developers with a large collection of image segmentation and registration algorithms, which are widely used for implementing custom software for specific medical imaging problems. ${ }^{53}$ Two ITK-based tools that gained popularity among researchers are ITK-SNAP for visualisation and segmentation of 3D images to aid in analysis of placental form and function calculations, and Elastix for image registration. ${ }^{5,54,55}$ Using Elastix, You et al. ${ }^{56}$ developed a semiautomated method for segmentation of the maternal and fetal compartments of the placenta using intravoxel incoherent motion. Finally, the large communitysupported 3D Slicer software with a user-friendly interface ${ }^{57}$ was used by Looney et al. ${ }^{58}$ to fully automate the segmentation of 3D placenta US data. 3D Slicer additionally supports registration, segmentation, and annotation, as well as scripting functionalities for batch-processing in Python. The design allows users to easily customise 3D Slicer by adding modules or extensions developed by the community to address specific imaging modalities or analysis tasks, such as studying fetal development or modelling placenta villi. ${ }^{59,60}$ SlicerMorph, an actively 
developed extension for 3D Slicer, provides biologists with the morphometric analysis tools for 3D volumetric and surface data, such as semilandmarking and Generalized Procrustes Analysis (GPA) for population-level analysis.

\section{CONCLUSION}

US is the gold standard for non-ionising noninvasive imaging used to assess placental structure. However, US alone is not sufficient to fully characterise placental pathophysiology, including vascular and metabolic functions and their relationship to clinical maternal and fetal outcomes. Emerging imaging modalities such as PAI are gaining popularity because they can be transparently integrated with existing imaging systems including US. Another useful clinical non-ionising modality discussed above in detail is MRI and its variants, including diffusionweighted, BOLD, and ASL, which have been studied to provide unique biomarkers to determine placental abnormalities. With respect to unravelling the role of metabolic heterogeneities in placenta health, several biomarkers have recently been discovered and studied using various technologies, such as ${ }^{1} \mathrm{H}-\mathrm{MRS}$ and Raman spectroscopy. Finally, to accurately quantify data generated by these imaging modalities, in terms of both 3D visualisation and multiple quantitative parameters, computer vision methods for quantitative image analysis are emerging. Various open-source platforms are being explored to develop analytical morphomics that can eventually predict effects on fetal growth based on dynamic changes in placental health and function. The recent explosion of image analysis and machine learning strategies will enhance both the quality and quantity of data available for making accurate diagnosis and monitoring therapeutics towards maintaining a healthy placenta. The clinical translation of these new frontiers is greatly needed to aid in the detection and diagnostic accuracy of placental abnormalities, reduce the risk of perinatal deaths, and improve the long-term course of offspring developmental health.

\section{References}

1. Pielesz A et al. FT Raman spectroscopy in the evaluation of biomarkers of normal and pathological placenta tissue. Mol Cell Biochem. 2019;458(1):125-32.

2. Maneas E et al. Photoacoustic imaging of the human placental vasculature. J Biophotonics. 2020;13(4):e201900167.

3. Aughwane R et al. Micro-CT and histological investigation of the spatial pattern of feto-placental vascular density. Placenta. 2019;88:36-43

4. Abramowicz JS, Sheiner E. Ultrasound of the placenta: a systematic approach. Part I: imaging. Placenta. 2008;29(3):225-40

5. Luo $\mathrm{J}$ et al. In vivo quantification of placental insufficiency by BOLD MRI: a human study. Sci Rep. 2017;7(1):3713.

6. Langheinrich $A C$ et al. Quantitative 3D micro-CT imaging of the human feto-placental vasculature in intrauterine growth restriction. Placenta. 2008;29(11):937-41.

7. Pratt $\mathrm{R}$ et al. Imaging the human placental microcirculation with micro-focus computed tomography: optimisation of tissue preparation and image acquisition. Placenta.
2017;60:36-9.

8. Lax A et al. The value of specific $M R I$ features in the evaluation of suspected placental invasion. J Magn Reson Imaging. 2007;25(1):87-93.

9. Teo $\mathrm{TH}$ et al. Use of magnetic resonance imaging in evaluation of placental invasion. Clin Radiol. 2009;64(5):511-6.

10. Rac MWF et al. Ultrasound predictors of placental invasion: the Placenta Accreta Index. Am J Obstet Gynecol. 2015;212(3):343.e1-7.

11. Wax IR et al. Second-trimester ultrasound-measured umbilical cord insertion-to-placental edge distance: determining an outcome-based threshold for identifying marginal cord insertions. J Ultrasound Med. 2020;39(2):351-8.

12. Ebbing $\mathrm{C}$ et al. Velamentous or marginal cord insertion and the risk of spontaneous preterm birth prelabor rupture of the membranes and anomalous cord length a populationbased study. Acta Obstet Gynecol Scand. 2017;96(1):78-85.

13. Coan PM et al. Developmental dynamics of the definitive mouse placenta assessed by stereology. Biol Reprod. 2004;70(6):1806-13.
14. Sørensen A et al. Changes in human placental oxygenation during maternal hyperoxia estimated by blood oxygen level-dependent magnetic resonance imaging (BOLD MRI). Ultrasound Obstet Gynecol. 2013;42(3):310-4.

15. Sørensen A et al. T2*-weighted placental MRI: basic research tool or emerging clinical test for placental dysfunction? Ultrasound Obstet Gynecol. 2019;55(3):293-302

16. Liu D et al. Human placenta blood flow during early gestation with pseudocontinuous arterial spin labeling MRI. J Magn Reson Imaging 2020;51(4):1247-57

17. Zun Z et al. Non-invasive placental perfusion imaging in pregnancies complicated by fetal heart disease using velocity-selective arterial spin labeled MRI. Sci Rep. 2017;7(1):16126.

18. Mervak BM et al. MRI in pregnancy: indications and practical considerations. J Magn Reson Imaging. 2019;49(3):621-31.

19. Roberts VHJ et al. Quantitative assessment of placental perfusion by contrast-enhanced ultrasound in macaques and human subjects. Am J Obstet Gynecol. 2016;214(3):369.e1-8.

20. Roberts VHJ et al. Early first 
trimester uteroplacental flow and the progressive disintegration of spiral artery plugs: new insights from contrast-enhanced ultrasound and tissue histopathology. Hum Reprod. 2017;32(12):2382-93.

21. Wu C, Bayer CL. Imaging placental function: current technology clinical needs and emerging modalities. Phys Med Biol. 2018;63(14):14TR01.

22. Kingdom JC et al. A placenta clinic approach to the diagnosis and management of fetal growth restriction. Am J Obstet Gynecol. 2018;218(2 Supplement):S803-17.

23. Kuc $\mathbf{S}$ et al. Evaluation of 7 serum biomarkers and uterine artery Doppler ultrasound for first-trimester prediction of preeclampsia: a systematic review. Obstet Gynecol Surv. 2011;66(4):225-39.

24. Li $L$ et al. Serum biomarkers combined with uterine artery Doppler in prediction of preeclampsia. Exp Ther Med. 2016;12(4):2515-20.

25. Alfirevic $Z$ et al. Fetal and umbilical Doppler ultrasound in normal pregnancy. Cochrane Database Syst Rev. 2015;2015(4):CD001450.

26. Calabuig-Navarro $\vee$ et al. Effect of maternal obesity on placental lipid metabolism. Endocrinology. 2017:158(8):2543-55.

27. Thomas MM et al. Oxidative stress impairs fatty acid oxidation and mitochondrial function in the term placenta. Reprod Sci. 2018;26(7):972

28. Austin C et al. Multielemental bioimaging of tissues in children's environmental health research. Curr Opin Pediatr. 2016;28(2):216-20.

29. Denison FC et al. Novel use of proton magnetic resonance spectroscopy (1HMRS) to non-invasively assess placental metabolism. PLoS One. 2012;7(8):e42926.

30. Song $\mathrm{F}$ et al. Assessment of the placenta in intrauterine growth restriction by diffusion-weighted imaging and proton magnetic resonance spectroscopy: a pilot study. Reprod Sci. 2016;24(4):575-81.

31. Chen S-J et al. Study of the molecular variation in pre-eclampsia placenta based on micro-Raman spectroscopy. Arch Gynecol Obstet. 2014;290(5):943-6.

32. Mallidi S et al. Photoacoustic imaging in cancer detection diagnosis and treatment guidance. Trends Biotechnol. 2011;29(5):213-21.

33. Bayer CL et al. Ultrasound-guided spectral photoacoustic imaging of hemoglobin oxygenation during development. Biomed Opt Express. 2017;8(2):757-63.

34. Lawrence DJ et al. Spectral photoacoustic imaging to estimate in vivo placental oxygenation during preeclampsia. Sci Rep. 2019;9(1):558.

35. Yamaleyeva LM et al. Photoacoustic imaging for in vivo quantification of placental oxygenation in mice. FASEB J. 2017;31(12):5520-9.

36. Yamaleyeva LM et al. Preclinical ultrasound-guided photoacoustic imaging of the placenta in normal and pathologic pregnancy. Mol Imaging. 2018;17:1536012118802721.

37. Yadav BK et al. A longitudinal study of placental perfusion using dynamic contrast enhanced magnetic resonance imaging in murine pregnancy. Placenta. 2016;43:90-7.

38. Krishnamurthy $U$ et al. Longitudinal changes in placental magnetic resonance imaging relaxation parameter in murine pregnancy: compartmental analysis. Gynecol Obstet Invest. 2016;81(3):193-201.

39. Stouffer RL, Woodruff TK. Nonhuman primates: a vital model for basic and applied research on female reproduction prenatal development and women's health. ILAR J. 2017;58(2):281-94

40. Schabel MC et al. Functional imaging of the nonhuman primate Placenta with endogenous blood oxygen leveldependent contrast. Magn Reson Med. 2016;76(5):1551-62.

41. Lo JO et al. Novel detection of placental insufficiency by magnetic resonance imaging in the nonhuman primate. Reprod Sci. 2018;25(1):64-73.

42. Avni R et al. Functional MRI of the placenta--from rodents to humans. Placenta. 2015;36(6):615-22.

43. Abramowicz JS, "The use of ultrasound contrast agents in placental imaging," Kay $\mathrm{HH}$ et al. (eds.), The Placenta, From Development to Disease (2011), Wiley \& Sons, pp.182-8.

44. Upputuri PK, Pramanik M. Recent advances in photoacoustic contrast agents for in vivo imaging. Wiley Interdiscip Rev Nanomed Nanobiotechnol. 2020 Jul;12(4):e1618.

45. Chen $\mathrm{C}-\mathrm{Y}$ et al. Evaluation of placental maturity by the sonographic textures. Arch Gynecol Obstet. 2011;284(1):13-8.

46. Do $Q N$ et al. Texture analysis of magnetic resonance images of the human placenta throughout gestation: a feasibility study. PLoS One. 2019;14(1):e0211060.
47. Romeo $\mathrm{V}$ et al. Machine learning analysis of MRI-derived texture features to predict placenta accreta spectrum in patients with placenta previa. Magn Reson Imaging. 2019;64:71-6.

48. Gupta $L$ et al. Segmentation of $2 D$ fetal ultrasound images by exploiting context information using conditional random fields. Conf Proc IEEE Eng Med Biol Soc. 2011;2011:7219-22.

49. Abramoff $M$ et al. Image Processing with ImageJ. Biophotonics Int. 2003;11:36-42.

50. Kidron D et al. Automated image analysis of placental villi and syncytial knots in histological sections. Placenta. 2017:53:113-8.

51. Bové $\mathrm{H}$ et al. Ambient black carbon particles reach the fetal side of human placenta. Nat Commun. 2019;10(1):3866.

52. Schindelin J et al. Fiji: an opensource platform for biological-image analysis. Nat Methods. 2012;9(7):676-82

53. Yoo $\mathrm{T}$ et al. Engineering and algorithm design for an image processing API: A technical report on ITK - the Insight Toolkit. Stud Health Technol Inform. 2002;85:586-92.

54. Yushkevich PA et al. User-guided 3D active contour segmentation of anatomical structures: significantly improved efficiency and reliability. Neurolmage. 2006;31(3):1116-28.

55. Klein S et al. elastix: a toolbox for intensity-based medical image registration. IEEE Trans Med Imaging. 2010;29(1):196-205.

56. You $W$ et al. Semi-automatic segmentation of the placenta into fetal and maternal compartments using intravoxel incoherent motion MRI. Proc SPIE Int Soc Opt Eng. 2017;10137: 1013726

57. Fedorov A et al. 3D Slicer as an image computing platform for the quantitative imaging network. Magn Reson Imaging. 2012;30(9):1323-41.

58. Looney $\mathrm{P}$ et al. Fully automated realtime 3D ultrasound segmentation to estimate first trimester placental volume using deep learning. $\mathrm{JCl}$ Insight. 2018;3(11):e120178.

59. Oyama $\mathrm{R}$ et al. Towards improved ultrasound-based analysis and 3D visualization of the fetal brain using the 3D Slicer. Ultrasound Obstet Gynecol. 2013;42(5):609-10.

60. Plitman Mayo R et al. Threedimensional modeling of human placental terminal villi. Placenta. 2016;43:54-60 